\title{
Sensitivity Analysis of Coupled Resonator Filters
}

\author{
Smain Amari, Member, IEEE
}

\begin{abstract}
Expressions for the sensitivity of coupled resonator filters with respect to the entries of the coupling matrix and the resistive terminations are presented. It is shown that the sensitivities of the network are all determined from a single analysis at a given frequency. The approach allows the elimination of the potentially time-consuming Monte Carlo analysis, which is used in tolerance analysis of this type of microwave structures. The technique can be applied to the sensitivity analysis of any linear system and is more general than the Adjoint Network Method.
\end{abstract}

Index Terms-Filters, linear systems, sensitivity, tolerance analysis.

\section{INTRODUCTION}

$\mathbf{M}$ ICROWAVE resonators are finding an increasing range of applications in modern communication systems such as bandpass filters with stringent requirements.

The computer-aided design of microwave resonator filters can be divided roughly into three steps. First, a network consisting of coupled lumped resonators is synthesized from the specifications on the frequency response. This step yields a coupling matrix which determines the amount of coupling between the different resonators and can be carried out using the theories presented in [1]-[5], for example. Second, models of physical resonators are used to attempt to reproduce the coupling matrix computed in the first step. Finally, a tolerance analysis to determine the sensitivity of the filter to manufacturing tolerances is performed.

Sensitivity analysis of microwave networks can be achieved using the concept of adjoint network as long as a network (and its adjoint) are available [6]. A limitation of the adjoint network approach stems from the fact that it assumes the validity of Kirchoff's laws. In more complex situations, such as encountered in modern high frequency circuits, the variables under consideration do not necessarily satisfy these laws. Such variables can be expansion coefficients in Moment Method solutions, or nodal values in the Finite Element Method; it is not obvious how the adjoint network method can be used or extended to these important situations.

Another popular approach in tolerance analysis is the Monte Carlo simulation, or similar statistical methods where the response of the filter is determined at randomly selected dimensions within the assumed errors to determine the robustness of the design. When a large number of variables are present, this approach can be time-consuming.

Manuscript received April 1999; revised May 2000. This paper was recommended by Associate Editor B. Leung.

The author is with the Department of Electrical and Computer Engineering, University of Victoria, Victoria, BC, V8W 3P6, Canada.

Publisher Item Identifier S 1057-7130(00)09321-6.
In this paper, we determine the sensitivity of resonator microwave filters directly from the matrix equations used in their analysis. More precisely, we are concerned with the sensitivity of the filter to variations in the entries of the coupling matrix. It is shown that the sensitivities of the response of the filter can be determined "analytically" from a single analysis of the network. From these sensitivities, acceptable bounds on the errors in the entries of the coupling matrix can be determined before an attempt is made to implement the network. Based on these results, the actual implementation can be either pursued or abandoned.

Although the technique is applied here only to the case of coupled resonator filters, it can be used to determine the sensitivities of any linear system whose response $[x]$ to an excitation $[e]$ is given by a linear equation of the form $[A][x]=[e]$ where $[A]$ is a linear operator representing the system.

\section{THEORY}

A typical structure considered in this paper is shown in Fig. 1. It consists of $N$ coupled lossless resonators. The resonant frequency is assumed to be unity. The frequency-independent coupling coefficient between resonators $i$ and $j$ is denoted by $M_{i j}=M_{j i}$. Possible shifts in the resonant frequencies of the resonators are included in the diagonal elements of the coupling matrix $[M]$. A voltage source of magnitude equal to unity and internal resistance $R_{1}$ excites the structure at resonator 1 . The load at the output is a resistor $R_{2}$ connected to resonator $N$. The loop currents in the different resonators are grouped in the vector $[I]$. The same model was used in [1].

Following the analysis in [1], the loop currents are governed by the following matrix equation:

$$
[\lambda-j R+M][I]=[A][I]=-j[e], \quad j^{2}=-1, \lambda=\omega-\frac{1}{\omega} .
$$

Here, $\lambda$ is the normalized frequency, $R$ is a matrix whose only nonzero entries are $R_{11}=R_{1}$, and $R_{N N}=R_{2}$ and $M$ are a symmetric square coupling matrix (with zero diagonal elements when only synchronously tuned resonators are considered). The excitation vector $[e]$ is given by $[e]^{t}=[1,0,0, \ldots, 0]$, where $t$ is the transposition operator. The discussion of the limitations of this model to narrow-band filters is well presented in [1] and is not repeated here.

The response functions we are interested in are the insertion and return loss of the filter as a function of frequency.

Using (1), we get the loop currents as

$$
[I]=-j\left[A^{-1}\right][e] .
$$




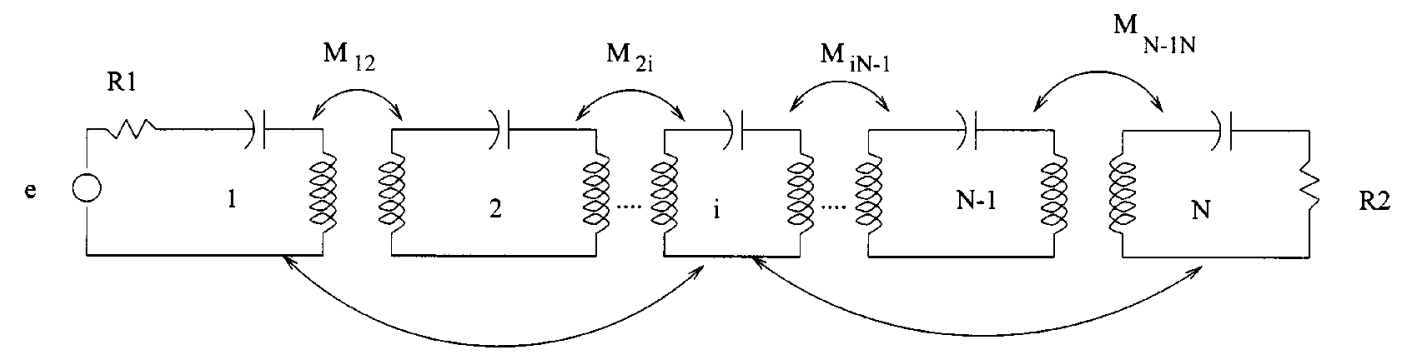

Fig. 1. Model of a general cross-coupled resonator bandpass filter.

From a simple analysis of the circuit, we get the following expressions for the transmission and reflection coefficients:

$$
\begin{aligned}
S_{21} & =2 \sqrt{R_{1} R_{2}} I_{N} \\
& =-2 j \sqrt{R_{1} R_{2}}\left[A^{-1}\right]_{N 1},
\end{aligned}
$$

and

$$
\begin{aligned}
S_{11} & =1-2 R_{1} I_{1} \\
& =1+2 j R_{1}\left[A^{-1}\right]_{11} .
\end{aligned}
$$

The coupling matrix and the termination resistances are assumed already determined using the theories in [1]-[5]. The basic question addressed in this paper is then the following: Given the coupling matrix $[M]$ and the resistive terminations $R_{1}$ and $R_{2}$, determine the sensitivities of $S_{11}$ and $S_{21}$ with respect to the entries of the matrix $[M]$ and the terminations. Obviously, it is possible to determine these sensitivities using finite differences whereby a separate analysis is carried out for each nonzero entry in the coupling matrix. Such a process can be time-consuming and may not be accurate unless exceedingly small increments are used. It is shown in this paper that these sensitivities can be determined from a single analysis, i.e., from the knowledge of the inverse of the matrix $[A]$ in (2).

Since the magnitude of the reflection and transmission coefficients can vanish for some frequency points, the definition of the sensitivity used is the unnormalized sensitivity defined by [6]

$$
S_{x}^{\left|s_{11}\right|}=\frac{\partial\left|S_{11}\right|}{\partial x}
$$

with a similar expression for $S_{21}^{x}$.

However, it can be easily shown that [7]

$$
\frac{\partial\left|S_{11}\right|}{\partial x}=\operatorname{Re}\left[\frac{\left|S_{11}\right|}{S_{11}} \frac{\partial S_{11}}{\partial x}\right], \quad \frac{\partial\left|S_{21}\right|}{\partial x}=\operatorname{Re}\left[\frac{\left|S_{21}\right|}{S_{21}} \frac{\partial S_{21}}{\partial x}\right]
$$

Using (3) and (4), we get

$$
\frac{\partial S_{11}}{\partial x}=-2 R_{1} \frac{\partial I_{1}}{\partial x}, \quad x \neq R_{1}
$$

and

$$
\frac{\partial S_{21}}{\partial x}=2 \sqrt{R_{1} R_{2}} \frac{\partial I_{N}}{\partial x}, \quad x \neq R_{1}, R_{2} .
$$

To calculate these derivatives, we take the derivative of the matrix equation $[I]=-j\left[A^{-1}\right][e]$ to get

$$
\frac{\partial[I]}{\partial x}=-j \frac{\partial\left[A^{-1}\right]}{\partial x}[e]-j\left[A^{-1}\right] \frac{\partial[e]}{\partial x}=-j \frac{\partial\left[A^{-1}\right]}{\partial x}[e] .(9)
$$

The last term is zero, since $[e]$ is a constant vector. Here, the derivative of a matrix is a matrix whose entries are the derivatives of the corresponding entries in the original matrix.

To calculate the derivative of the inverse of the matrix $[A]$, we take the derivative of the identity $[A]\left[A^{-1}\right]=[U]$, where $[U]$ is the identity matrix, to get

$$
\frac{\partial\left[A^{-1}\right]}{\partial x}=-\left[A^{-1}\right] \frac{\partial[A]}{\partial x}\left[A^{-1}\right] .
$$

Combining (9) and (10), we get

$$
\frac{\partial[I]}{\partial x}=j\left[A^{-1}\right] \frac{\partial[A]}{\partial x}\left[A^{-1}\right][e] .
$$

To proceed further, we need to introduce a few notations. Let us define the topology matrix of the network by $P_{i j}=1$ if $M_{i j} \neq 0$, and $P_{i j}=0$ if $M_{i j}=0$.

When the variable $x$ is replaced by an element of the coupling matrix $M_{p q}$ in (11), which is then used in (7) and (8), we get the simple results

$$
\frac{\partial S_{11}}{\partial M_{p q}}=-4 j R_{1} P_{p q}\left[A^{-1}\right]_{p 1}\left[A^{-1}\right]_{q 1}
$$

and

$$
\begin{aligned}
\frac{\partial S_{21}}{\partial M_{p q}}= & 2 j \sqrt{R_{1} R_{2}} P_{p q} \\
& \cdot\left(\left[A^{-1}\right]_{N p}\left[A^{-1}\right]_{q 1}+\left[A^{-1}\right]_{N q}\left[A^{-1}\right]_{p 1}\right) .
\end{aligned}
$$

Here, the symmetry of the matrices $[A]$ and $\left[A^{-1}\right]$ was used.

The partial derivatives of the reflection and transmission coefficients with respect to the resistive loads can be determined similarly

$$
\begin{aligned}
& \frac{\partial S_{11}}{\partial R_{1}}=2 j\left[A^{-1}\right]_{11}-2 R_{1}\left[A^{-1}\right]_{11}\left[A^{-1}\right]_{11} \\
& \frac{\partial S_{11}}{\partial R_{2}}=-2 R_{1}\left[A^{-1}\right]_{1 N}\left[A^{-1}\right]_{N 1} \\
& \frac{\partial S_{21}}{\partial R_{1}}=-j \sqrt{\frac{R_{2}}{R_{1}}}\left[A^{-1}\right]_{N 1}+2 \sqrt{R_{1} R_{2}}\left[A^{-1}\right]_{N 1}\left[A^{-1}\right]_{11}
\end{aligned}
$$




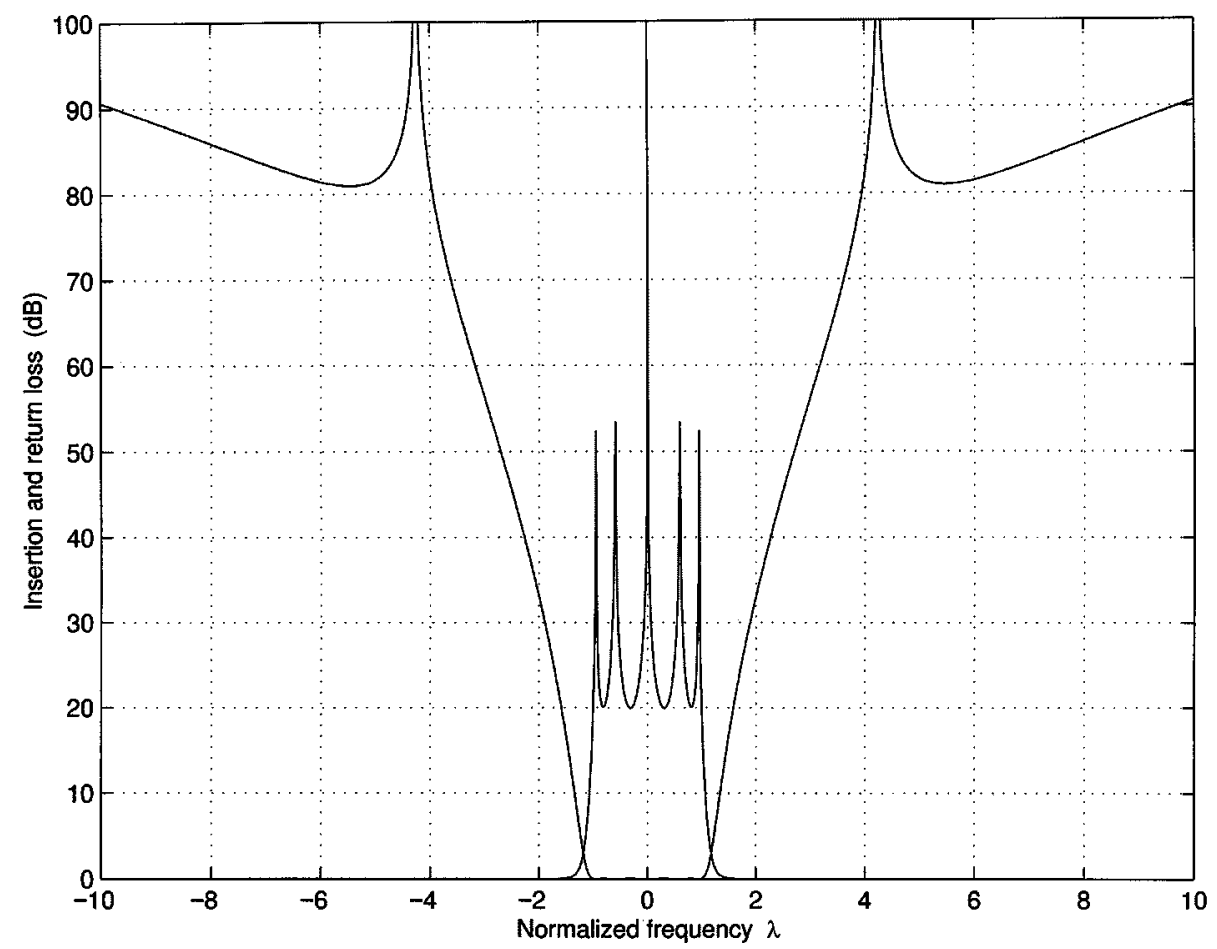

Fig. 2. Insertion and return loss (in decibels) of a 5-pole pseudo-elliptic filter [9].

TABLE I

COUPLING MATRIX OF A FIVE-RESONATOR FILTER, $R=20 \mathrm{~dB}, R_{1}=R_{2}=1.025$

\begin{tabular}{c|c|c|c|c|c}
\hline \hline $\mathrm{ij}$ & 12 & 23 & 34 & 45 & 14 \\
\hline$M_{i j}$ & -0.863 & -0.647 & -0.632 & -0.863 & 0.020 \\
\hline$[9]$ & -0.863 & -0.647 & -0.632 & -0.863 & 0.020 \\
\hline
\end{tabular}

and

$$
\frac{\partial S_{21}}{\partial R_{2}}=-j \sqrt{\frac{R_{1}}{R_{2}}}\left[A^{-1}\right]_{N 1}+2 \sqrt{R_{1} R_{2}}\left[A^{-1}\right]_{N N}\left[A^{-1}\right]_{N 1} .
$$

To obtain the final expressions of the sensitivities, appropriate combinations of (3), (4), and (12)-(17) are to be used in (6).

It has been brought to the attention of the author that Zaki and Atia presented sensitivity analysis of coupled resonator filters [8]. Their results are, however, given without derivation. In contrast, the derivation presented here can be used to establish the sensitivities of any linear system.

\section{NUMERICAL RESULTS}

The theory presented here is applied to the sensitivity analysis of a fifth-order pseudo-elliptic filter with two transmission zeros, one on each side of the passband. The response of the low-pass prototype is shown in Fig. 2. The passband return loss is $20 \mathrm{~dB}$ and the transmission zeros are located at $\lambda= \pm 4.25$. This prototype can be obtained from the coupling matrix given in Table I. Note that these values agree very well with those given in reference [9] for the same prototype.
The sensitivities of the reflection and transmission coefficients with respect to the entries of the coupling matrix and the resistive loads were determined from the present theory as well as finite differences, and showed perfect agreement.

Fig. 3 shows the quantity $\partial\left|S_{11}\right| / \partial R_{1}$ (solid line) and $\partial\left|S_{11}\right| / \partial R_{2}$ (dashed line) as a function of frequency. The frequency range was limited to approximately the passband since $\left|S_{11}\right|$ and its derivatives decrease rapidly for frequencies outside of this range. It is interesting to observe that the reflection coefficient is equally sensitive to variations in the two resistive terminations. This result implies that, to first order, the reflection coefficient is not affected by a positive (small) error in $R_{1}$ and the opposite error in $R_{2}$. That this is the case is shown in Fig. 4. A $10 \%$ error was used in both $R_{1}$ and $R_{2}$ once with the same sign (dashed-dotted line, $-10 \%$ ) and once with the opposite sign (dashed line, $\Delta R_{1}=+10 \%$, $\left.\Delta R_{2}=-10 \%\right)$. The solid line is the return loss with the exact values of $R_{1}$ and $R_{2}$. This example shows that, as far as the in-band return loss is concerned, opposite errors in the resistive terminations have little effect on the performance of the filter.

The sensitivities of the insertion loss to $M_{14}$ (solid line) and $M_{12}$ were was also determined and are shown in Fig. 5. Since the cross coupling term $M_{14}$ is responsible for the presence of the transmission zeros, it is not surprising that the stopband insertion loss of the filter is more sensitive to $M_{14}$ than $M_{12}$. To a first approximation, errors in $M_{14}$ determine the robustness of the filter in the stopband, especially in the vicinity of the transmission zeros.

The sensitivities of the reflection coefficient with respect to the entries of the coupling matrix were also determined and are 


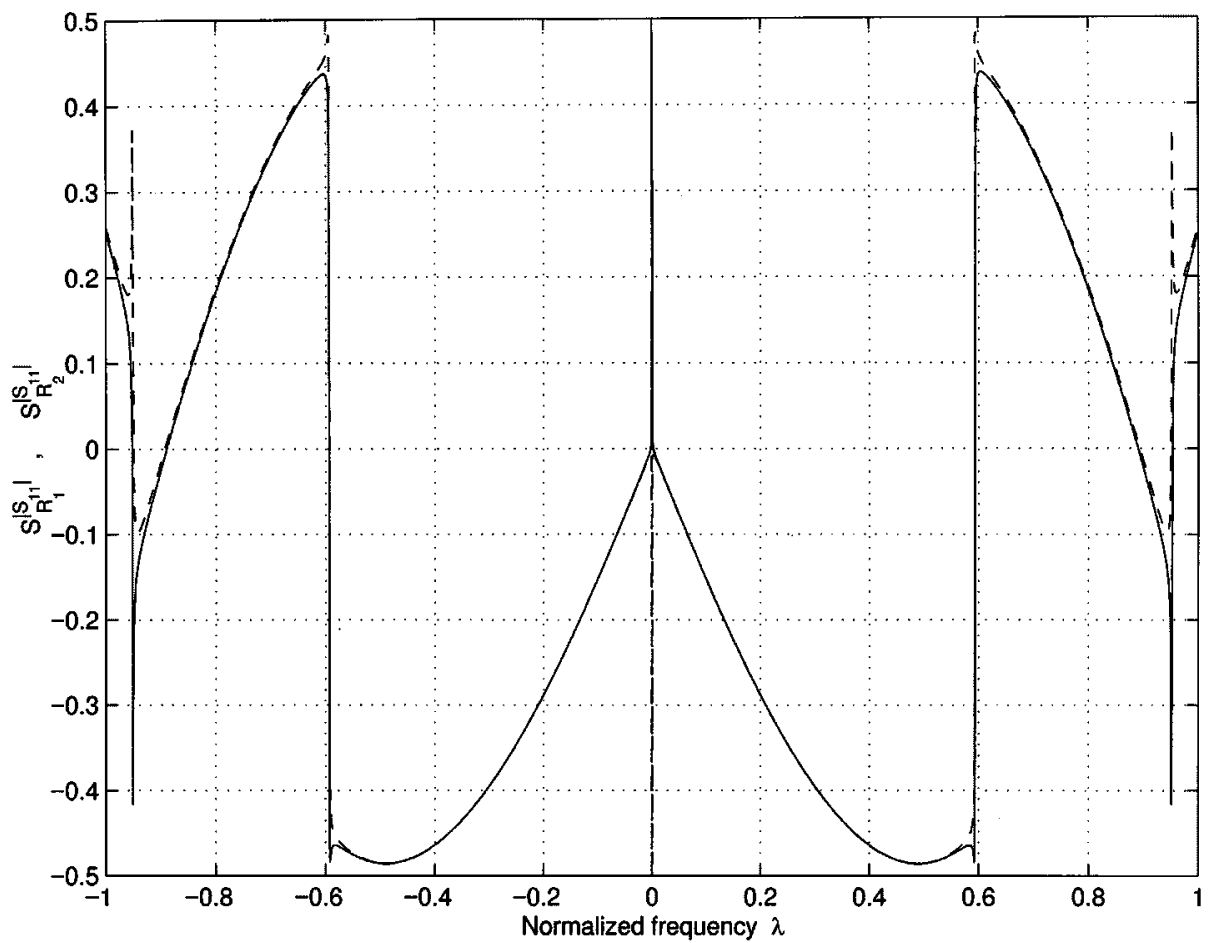

Fig. 3. Unnormalized sensitivities of $\left|S_{11}\right|$ versus $R_{1}$ and $R_{2}$.

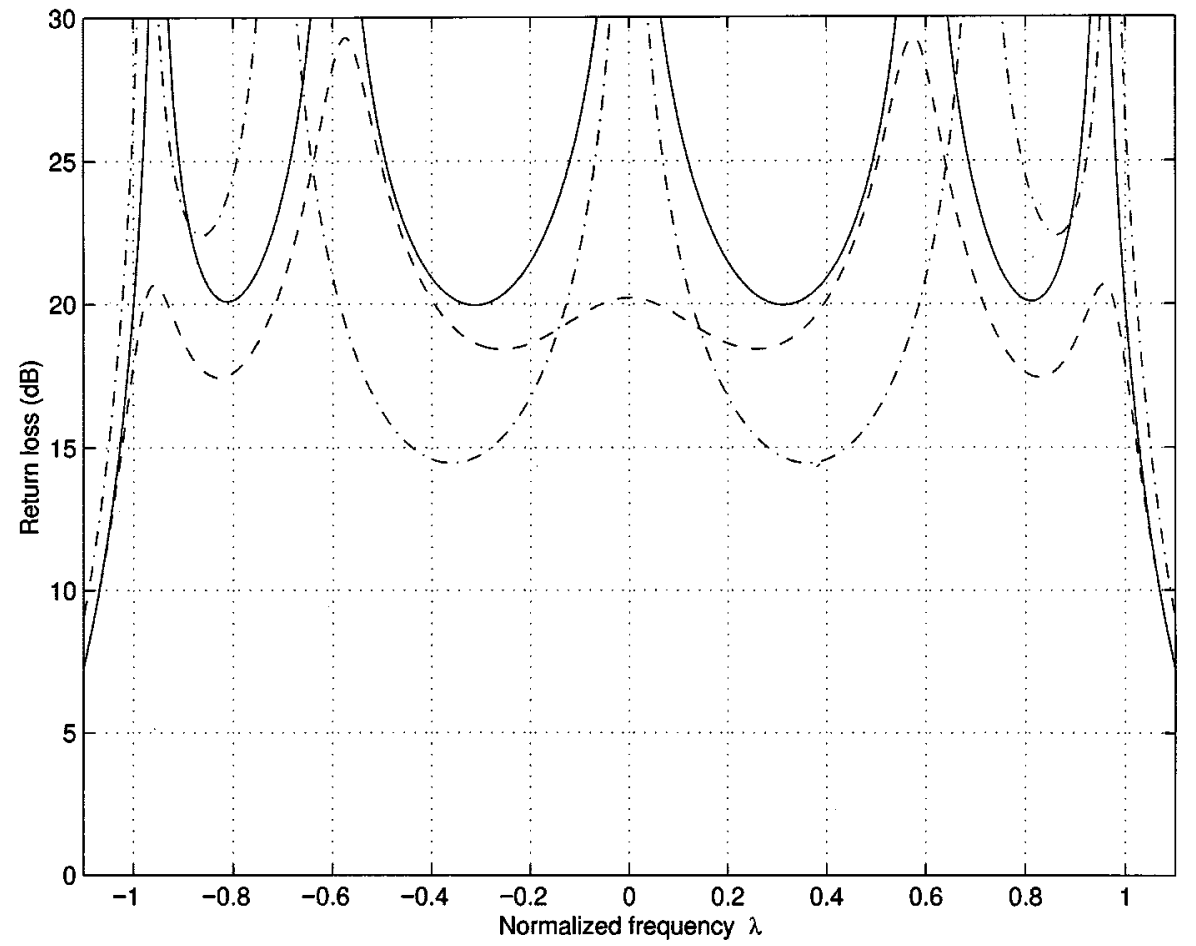

Fig. 4. Return loss (in decibels) for $\Delta R_{1}=\Delta R_{2}=0$ (solid line), $\Delta R_{1}=+10 \%, \Delta R_{2}=-10 \%$ (dashed line), and $\Delta R_{1}=\Delta R_{2}=-10 \%$ (dashed-dotted line).

shown in Fig. 6. The discontinuities which occur at the zeros of $\left|S_{11}\right|$ are typical of the absolute value function. From the relative signs and magnitudes of these sensitivities, and those of the transmission coefficient, the tuning of the filter can be decided and possibly accelerated. The sensitivities can also be used to determine the "worst" performance of the filter where all errors contribute the same sign to the response. Although such a result can be obtained from a potentially lengthy statistical analysis, the analytic formulas presented in this paper allows one to decide beforehand on the robustness of the filter. 


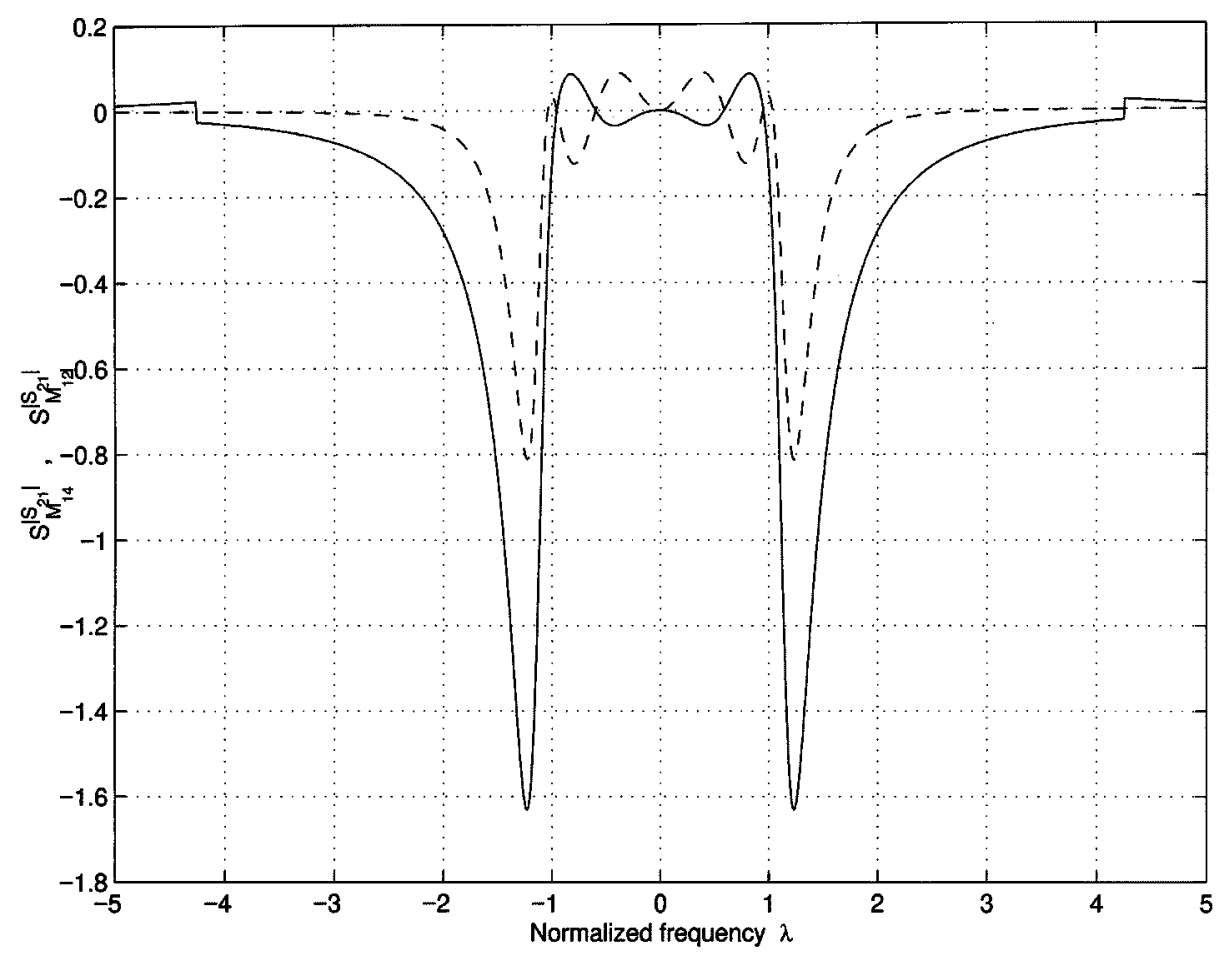

Fig. 5. Unnormalized sensitivities of $\left|S_{21}\right|$ versus $M_{14}$ (solid line), $M_{12}$ (dashed line).

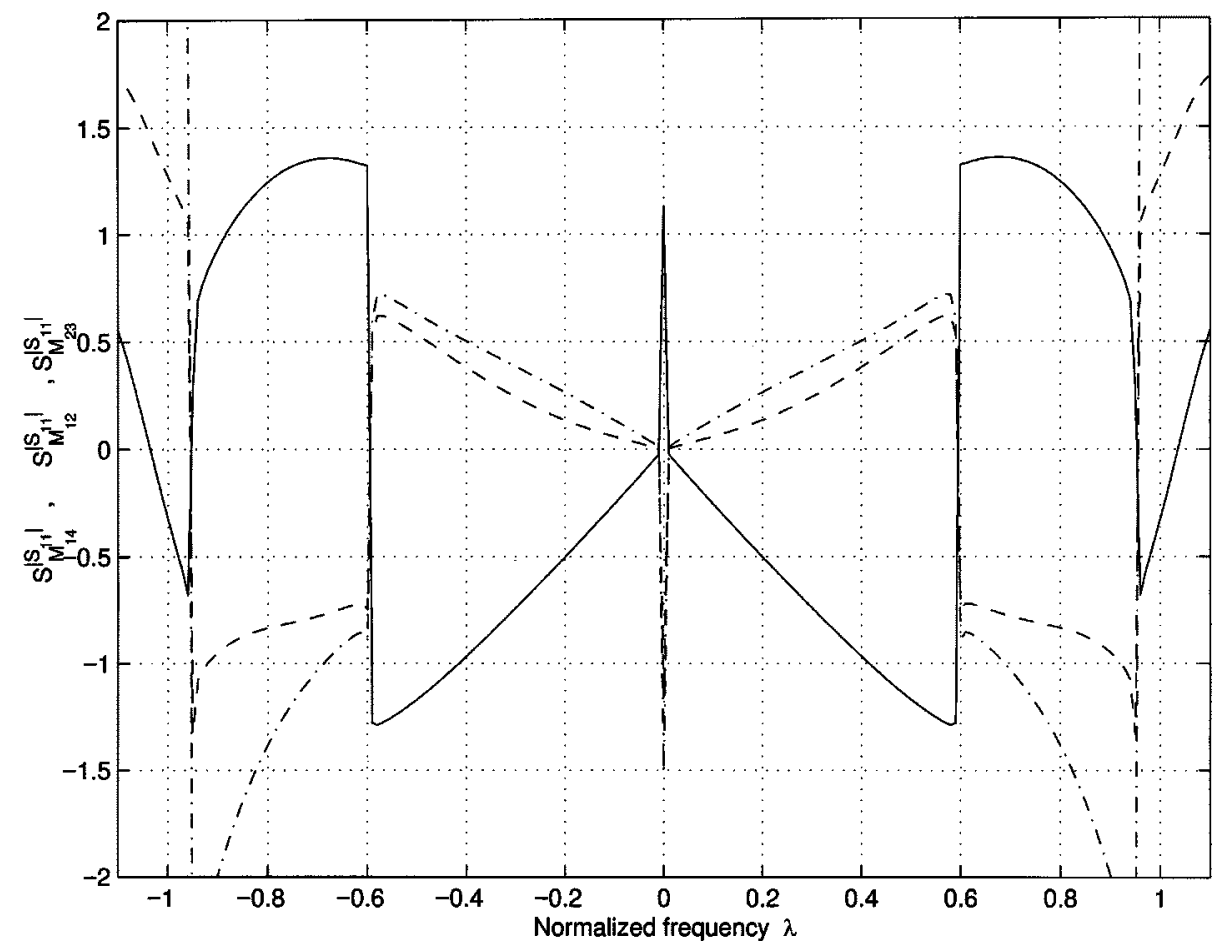

Fig. 6. Unnormalized sensitivities of $\left|S_{11}\right|$ versus $M_{12}$ (solid line), $M_{14}$ (dashed line) and $M_{23}$ (dashed-dotted line).

\section{CONCLUSION}

Analytical expressions for the sensitivities of the reflection and transmission coefficients of coupled resonator filters were presented. All sensitivities are obtained from a single analysis of the structure. The signs and magnitudes of these sensitivities can be used in determining the robustness of the synthesized filter beforehand. The obtained sensitivities agree well with those ob- 
tained from finite differencing (not shown here). The technique can be used to establish the sensitivities of any linear system and is more general than the Adjoint Network Method.

\section{REFERENCES}

[1] A. Atia and A. E. Williams, "New type of waveguide bandpass filters for satellite transponders," COMSAT Tech. Rev., vol. 1, no. 1, pp. 21-43, 1971.

[2] A. E. Atia, A. E. Williams, and R. W. Newcomb, "Narrow-band multiple-coupled cavity synthesis," IEEE Trans. Circuits Syst., vol. CAS-21, pp. 649-655, Sept. 1974.

[3] R. J. Cameron, "General prototype network synthesis methods for microwave filters," ESA J., vol. 6, pp. 193-206, 1982.

[4] R. J. Cameron and J. D. Rhodes, "Asymmetric realizations of dual-mode bandpass filters," IEEE Trans. Microwave Theroy Tech., vol. MTT-29, pp. 51-58, Jan. 1981.

[5] IEEE Trans. Microwave Theory Tech.-Special Issue, vol. MTT-30, Sept. 1982.

[6] L. O. Chua and P.-M. Lin, Computer-Aided Analysis of Electronic Circuits. Englewood Cliffs: Prentice-Hall, 1975.

[7] K. C. Gupta, R. Garg, and R. Chadha, Computer Aided Design of Microwave Circuits. Norwood, MA: Artech House, 1981.
[8] K. A. Zaki and A. E. Atia, "Sensitivity analysis of multi-coupled cavity filters," in IEEE Int. Symp. Circuits and Systems, May 1978, pp. 790-793.

[9] U. Rosenberg, "New 'planar' waveguide cavity elliptic function filters," Eur. Microwave Conf. Dig., pp. 524-527, 1995.

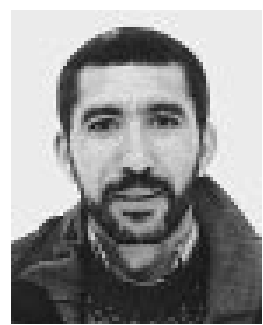

Smain Amari (M'98) received the D.E.S. degree in physics and electronics from Constantine University, Constantine, Algeria, in 1985, the M.S. degree in electrical engineering and the Ph.D. degree in physics, both from Washington University, St. Louis, in 1989 and 1994, respectively.

$\mathrm{He}$ is currently a Research Associate and Adjunct Assistant Professor with the Department of Electronics and Computer Engineering, University of Victoria, Victoria, BC, Canada. His interests include numerical methods in electromagnetics, numerical analysis, applied mathematics, wireless and optical communications, applied physics, and application of quantum field theory in quantum many-particle systems. 